
DE FLUIDOS COMBUSTÍVEIS SINTÉTICOS

\title{
CONTRIBUTIONS OF THE NON-EUCLIDEAN GEOMETRY ON THE PRODUCTION OF SYNTHETIC LIQUID FUELS
}

\author{
DE BONI, Luis Alcides Brandini'; SILVA, Isaac Newton Lima da'. \\ ${ }^{1}$ Pontifícia Universidade Católica do Rio Grande do Sul, Pós-Graduação em Engenharia e Tecnologia de \\ Materiais. Av. Ipiranga, 6681 - Partenon - Porto Alegre/RS - CEP: 90619-900, Brasil \\ ${ }^{*}$ Autor correspondente e-mail: labdeboni@gmail.com
}

\section{RESUMO}

As necessidades de produzirem combustíveis a partir de fontes menos poluentes, a partir de matériasprimas renováveis, consumindo menos energia e gerando menos resíduos são algumas das prioridades para garantir o futuro sustentável da nação. Neste contexto determinar adequadamente quando a reação química que produz o combustível atingiu o seu fim é importante para elevar a eficiência energética do processo. A técnica utilizada para monitorar a reação de produção do combustível consistiu em emitir um feixe de laser no meio reacional, produzindo um holograma. A medida da taxa de variação de tal holograma em função da cinética da reação permite determinar quando a reação química entrou em equilíbrio químico. Como resultado, é possível correlacionar a variação do espalhamento da luz com a cinética da reação.

Palavras-chave: Transesterificação, Geometria não Euclidiana, Equilíbrio Químico, Matemática Aplicada.

\begin{abstract}
The requirements for producing fuels from less polluting sources, from renewable raw materials, consuming less power and to generate a smaller amount of waste are some of the priorities to ensure the sustainable future of the nation. In this context, correctly determine when the chemical reaction that produces the renewable fuel has reached the chemical balance is important to increase the energetic efficiency. The technique used to monitor the reaction that produces the fuel consisted in to deliver a laser beam in the reaction medium, producing a hologram. The measure of the rate of change of the hologram surface as a function of the reaction kinetics, allows to determine when the chemical reaction came to chemical equilibrium. As result it is possible to correlate the light scattering variation with the reaction kinetics.
\end{abstract}

Key-words: Transesterification, non-Euclidean geometry, Chemical Equilibrium, Applied Mathematics 


\section{INTRODUÇÃO}

A produção de fluídos combustíveis sintéticos, de origem renovável, é tida como prioridade estratégica para o desenvolvimento nacional (MCT, 2008; MD, 2010; $4^{\mathrm{a} C N C T I, ~}$ 2010). Um dos combustíveis geralmente apresentados como portador de futuro é o biodiesel.

Neste trabalho serão exploradas questões fundamentais para o desenvolvimento da tecnologia de produção do biodiesel, como por exemplo, a determinação acurada do momento que a reação de transesterificação atingiu o estado de equilíbrio químico. Em geral as técnicas de acompanhamento são dispendiosas, como por exemplo, a cromatografia. É fundamental a procura por técnicas de baixo custo e de rápida resposta.

Quando pensamos na produção de um combustível renovável, associamos a esta idéia conceitos intrinsecamente provindos da química verde (LENARDÃO, 2002), como o uso de fontes renováveis de matérias-primas, busca pela eficiência energética, economia de átomos e análise em tempo real para a prevenção da poluição. Há o desafio de como prover estas soluções da melhor forma possível.

O biodiesel é um combustível produzido com o uso de fontes renováveis de matériasprimas, geralmente através da reação de transesterificação, conforme a Figura 1. Nesta reação química uma molécula de um triglicerídio qualquer é convertida no respectivo monoalquiléster.

De acordo com Van Gerpen (2002) a transesterificação utilizando óleo de soja, metanol e $\mathrm{NaOH}$, demora um período de tempo para a reação ser concluída. Este tempo varia em função da temperatura reacional. $\mathrm{A} 20^{\circ} \mathrm{C}$, por exemplo, a reação será concluída entre 4 e 8 horas e a $60^{\circ} \mathrm{C}$ a reação será concluída entre 1 e 2 horas. A diferença, além do tempo de reação, é o custo energético da reação. A Figura 2 apresenta a influência da temperatura sobre o tempo de reação.

O intervalo de tempo, entre 1 e 2 horas, apresenta um consumo energético diferente. Desta forma, determinar rapidamente quando a reação entrou em equilíbrio químico, ou foi concluída, é importante para melhorar a eficiência energética da reação.
INFLUÊNCIA DA TEMPERATURA SOBRE O TEMPO DA REAÇÃO DE TRANSESTERIFICAÇÃO

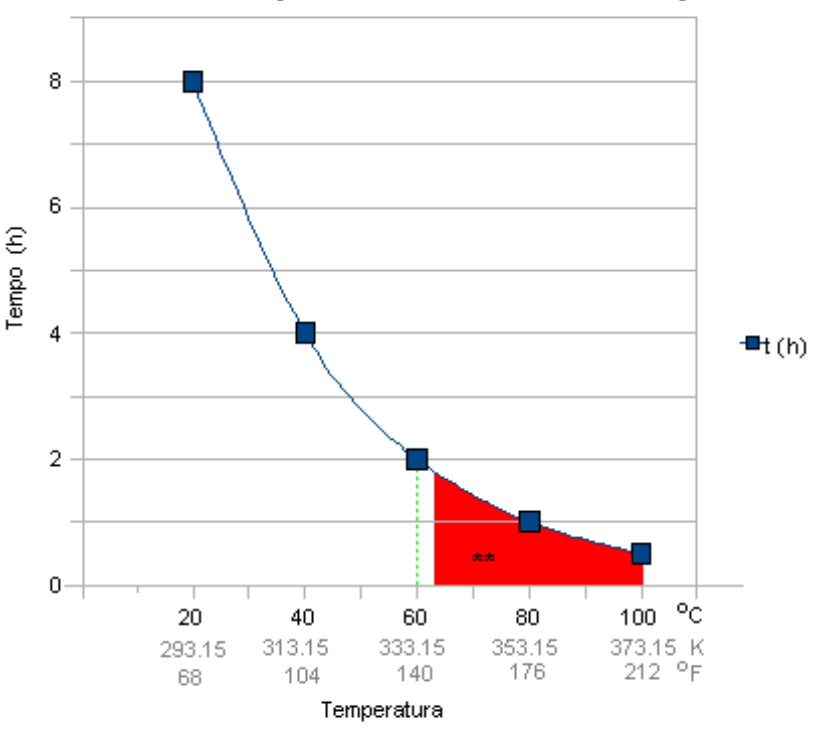

* Utilizando-se metanol e $\mathrm{NaOH}$

*t Deve ser utilizado um reator adequado para trabalhar nestas temperaturas

Figura 2. Tempo de reação em função da temperatura.

Fonte: TchêQuímica (2008), adaptado de Van Gerpen (2007)

Ao refletir sobre isto, naturalmente surgem as questões: "o que a geometria tem haver com isto?" e "o que é geometria não Euclidiana?".

Para responder a estas questões analisaremos inicialmente o que é a geometria Euclidiana. A geometria Euclidiana, de forma simplista, pode ser resumida pela matemática ensinada na escola. Esta geometria baseia-se em 5 postulados (FITZPATRICK, 2010):

1. Uma linha reta pode ser traçada entre dois pontos quaisquer;

2. Uma reta pode ser estendida indefinidamente para os dois lados;

3. Dado um centro e um raio, é sempre possível traçar um círculo;

4. Todos os ângulos retos são iguais entre $\mathrm{si}$;

5. Se duas retas $(A$ e $B)$ em um plano são interceptadas por outra reta $C$ tal que os ângulos ( $a$ e b) de um mesmo lado de $C$ somam um valor menor que $180^{\circ}$, então as retas $A$ e $B$, quando prolongadas do lado dos ângulos a e b, irão se encontrar 
em algum ponto.

Não é dubitável a veracidade dos 4 primeiros postulados, porém o 5 postulado nem sempre é verdadeiro.

De acordo com os livros didáticos de matemática tradicionalmente utilizados no ensino escolar Brasileiro, tem-se que a soma dos ângulos internos de um triângulo é sempre igual a $180^{\circ}$, todavia isto não é real. A soma dos ângulos internos de um triângulo só será igual se a curvatura Gaussiana da superfície $(\mathrm{K})$, onde o triângulo esta exposto for igual a zero (Bonola, 1912).

Neste trabalho será utilizado o conceito de linha reta definido por Georg Friedrich Bernhard Riemann (1826 - 1866). "Uma linha reta entre dois pontos é apenas a curva de menor comprimento, sobre a superfície, entre os dois pontos." (Gray, 1988).

No mundo natural, $\mathrm{K}$ pode ser maior, menor ou até igual a zero. Isto implica dizer que a soma dos ângulos internos de um triângulo pode ser maior, menor ou igual a $180^{\circ}$.

Neste contexto será analisado o resultado de imagens digitalizadas da reação de transesterificação monitoradas por espectroscopia de correlação de fótons em tempo real.

Para comentar os efeitos da digitalização do experimento, gostaria de fazer minhas as palavras de Klügel (1763) (Volkert, 2006) apud O'Shea 2009, "Evidentemente, talvez seja possível que retas que não se cruzem, talvez divirjam uma da outra. Sabemos que isto é absurdo, não por inferência rigorosa ou por conceitos claros de linhas retas e curvas, mas pela experiência e julgamento de nossos olhos".

\section{DESENVOLVIMENTO}

Uma reação de transesterificação foi montada conforme a Figura 3 . Onde um feixe de laser foi emitido a partir de uma fonte luminosa (1), atravessou um meio reacional (3) e impressionou um sensor (4), conforme apresentado por Silva e De Boni (2010).

A Figura 4 é uma montagem de uma imagem que foi capturada do experimento, onde se observa o holograma original e duas cópias da imagem com as bordas destacadas. Considerando-se a imagem central, o destaque da borda é feito com uma reta no sentido
Euclidiano, onde a curvatura gaussiana $(\mathrm{K})$ é igual a zero. O sólido de revolução desta reta gera um cone. $\mathrm{E}$ a imagem mais a direita, da Figura 4, utiliza a visão de reta proposta por Riemann, o sólido de revolução desta reta gera uma figura conhecida como pseudo-esfera (Figura 5), tal figura também é referida como tractróide, tractricóide, tractrizóide ou antiesfera, (Steinhaus, 1999). A pseudo-esfera é uma superfície de revolução, de curvatura gaussiana negativa constante $(K<0)$, gerada através da rotação de uma tractriz sobre sua assíntota.

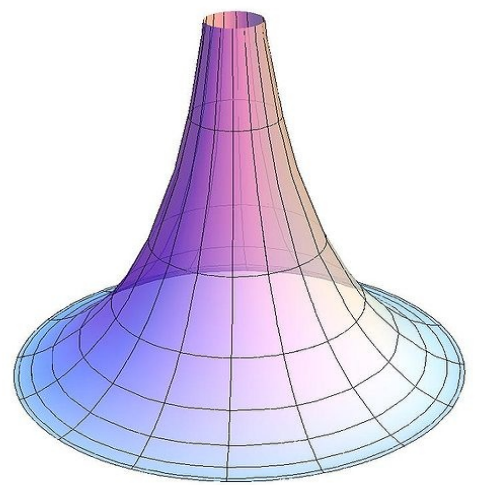

Figura 5. Pseudo-esfera

Fonte: wikimidia (2010)

Durante $\mathrm{o}$ andamento da reação de transesterificação, a superfície ou o volume do holograma da Figura 4 variou de magnitude. Esta superfície parou de variar seu tamanho quando a reação supostamente entrou em equilíbrio químico, conforme a seqüência de imagens da Figura 6.

O monitoramento da reação de transesterificação foi dividido em quatro conjuntos de instantes específicos A, B, C e D. Estes instantes possuíam características bem distintas, e podiam ser observados à olho nu, todavia o uso do equipamento de proteção individual foi realizado, para evitar danos à visão. Os instantes são descritos a seguir:

Instante $\mathbf{A}$ : antes do início da reação. Antes de a reação iniciar foi capturado o valor da intensidade luminosa impressa pelo laser sobre o sensor LDR. Desta forma foi possível determinar a absorvância que uma camada de óleo de soja de $10 \mathrm{~cm}$ de espessura, à $60^{\circ} \mathrm{C}$ ofereceu a passagem do feixe de laser. Este valor foi arbitrado como $T=0 \mathrm{~s}$. $\mathrm{O}$ efeito visual de $\mathrm{T}=0 \mathrm{~s}$ pode ser analisado na Figura 6 , sob o índice de número 1 , da série de fotografias. 
Instante B: após a adição da solução de alcoólica de catalisador. Ocorreram uma série de eventos muito velozes, iniciados no momento em que foi adicionado ao reator a mistura de álcool e catalisador. Estes eventos causaram grande perturbação ou dispersão na trajetória do laser. Na Figura 6, estes eventos estão sob os índices de 2 à 10. Dentro do reator, o feixe de laser disperso tomava a forma de um cone ou de uma pseudo-esfera, e ao sair do reator, a forma parecia se alterar para um cilindro. Tal fenômeno, curiosamente, lembrou de forma distante a difração cônica interna da luz em um prisma com simetria biaxial, conforme o descrito por Hamilton (1837) em Third supplement to an essay on the theory of systems of rays.

Instante C: após os instantes de grande dispersão do feixe de laser. A mistura de óleo de soja, metanol e catalisador tornou-se homogênea, e foi monitorado um fenômeno de aumento de intensidade luminosa sobre o sensor LDR (Light Dependent Resistor), caracterizado pela redução da dispersão (ou absorção) do feixe de laser. Estes instantes estão representados na Figura 6 , sob os índices de 11 a 21 . O aumento da intensidade luminosa sobre o sensor LDR foi registrado através da redução da resistência elétrica do sensor, até um instante em que a resistência e a dispersão do feixe de laser se tornaram mínimas e a passagem do feixe foi a mais elevada durante o andamento da reação (Figura 6, índice 21).

Instante D: Após a dispersão mínima do feixe de laser e menor quantidade de resistência elétrica obtida através do sensor LDR. Após este Instante $(C)$, voltou a ser observado e registrado a elevação da resistência elétrica no sensor LDR e um novo aumento da dispersão do feixe de laser, porém menos intenso que a dispersão provocada pela adição do álcool e do catalisador. Na Figura 6, estes eventos estão registrados sob os índices de 22 à 25.

\section{RESULTADOS E DISCUSSÃO:}

Tendo em vista a dificuldade de se determinar experimentalmente a curvatura gaussiana $(K)$ da superfície observada, tem-se que:

$\rightarrow \mathrm{K}=0$. A figura pode ser descrita como um cone. Desta forma, a soma dos ângulos $(\angle)$ internos do triângulo formado pela superfície contida na Figura 4 será: $\angle \mathrm{A}+\angle \mathrm{B}+\angle \mathrm{C}=\pi$. $\mathrm{O}$ volume interno desta figura pode ser calculado pela expressão $1 / 3 \cdot \pi \cdot h \cdot r^{2}$.

Também, $\rightarrow K=0$, a cinética da reação não pode ser monitorada pela variação da soma dos ângulos internos do triângulo, pois $\angle \mathrm{A}+$ $\angle \mathrm{B}+\angle \mathrm{C}=\pi=$ cte.

Porém, $\rightarrow K \neq 0$. A figura não pode ser descrita como um cone, devendo ser descrita como uma pseudo-esfera. Assim sendo:

$\angle \mathrm{A}+\angle \mathrm{B}+\angle \mathrm{C} \neq \pi$. $\mathrm{E}$ o volume interno desta figura pode ser calculado pela expressão $2 / 3$. $\pi \cdot r^{3}$, se o limite de $r$ tender ao infinito.

Então, $\rightarrow \mathrm{K} \neq 0$, a cinética da reação pode ser monitorada através variação da soma dos ângulos internos de um triângulo, pois $\angle A+\angle B$ $+\angle \mathrm{C} \neq \pi \neq$ cte.

Determinar a curvatura da superfície obtida experimentalmente é importante para ir além da análise em tempo real da reação de transesterificação para a prevenção da poluição, isto permitirá a predição com maior precisão do momento em que a reação irá entrar em equilíbrio químico, uma vez que serão utilizadas as equações que melhor descrevem o holograma contido na Figura 4.

\section{CONCLUSÕES:}

Através deste trabalho conclui-se que é possível determinar em tempo real o momento aproximado em que a reação de transesterificação do óleo de soja com metanol atinge o estado de equilíbrio químico, através de técnicas de geometria e/ou trigonometria.

Apesar de incomum, e ainda em estágios de desenvolvimento, a técnica produz resultados adequados e com boa reprodutibilidade. Esta metodologia tende a elevar a sustentabilidade dos processos químicos.

\section{AGRADECIMENTOS:}

O autor agradece a PETROBRAS e ao $\mathrm{CNPq}$ pelo suporte financeiro fornecido a esta pesquisa.

\section{REFERÊNCIAS:}

1. MCT - Ministério da Ciência e Tecnologia. Prioridade Estratégica III - Pesquisa, Desenvolvimento e Inovação em Áreas 
Estratégicas.

Disponível

em:<http://www.mct.gov.br

/upd_blob/0021/21593.pdf>. Acesso em: ago. 2008.

2. MD - Ministério da Defesa. Tecnologias de Interesse da Defesa Nacional. Disponível em: <https://www.defesa .gov.br/ciencia_tecnologia/links_ass_acad e_1.htm>. Acesso em: ago. 2008.

3. $\mathrm{CNCTI}$ - Conferência Nacional de Ciência, Tecnologia e Inovação. Disponível em: <http://cncti4.cgee.org .br/>. Acesso em: Dez. 2010.

4. LENARDÃO, E. J., FREITAG, R. A., DABDOUB, M. J., BATISTA, C. F., SILVEIRA, C.C. "Green Chemistry" Os 12 princípios da química verde e sua inserção nas atividades de ensino e pesquisa. Química Nova. v. 26, n. 1, p. 123-129, 2003.

5. FITZPATRICK, Richard. Euclid's Elements of Geometry. Disponível em: $<$ http://farside.ph.utexas.edu/euclid/Eleme nts.pdf>. Acesso em Mai. 2010.

6. BONOLA, Roberto. Non-Euclidean Geometry. A Critical and Historical Study of it's Development. Chicago: The open Court Publishing Company, 1912. 290p.

7. VOLKERT, Klaus. Georg Klügel's dissertation (1763) now accessible on the worldwide web. Disponível em $<$ http://www.sciencedirect .com/science? ob=ArticleURL\&_udi=B6WG9-4K1X874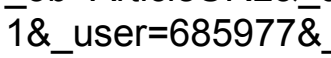 coverDate $=08 \% 2 \mathrm{~F} 31 \% 2 \mathrm{~F} 2006 \&$ rdoc $=1 \&$ fmt=high\&_orig=search\&_origin=search \&_sort $=d \& \_$docanchor $=\&$ view $=c \&$ \& search Strld $=1509589375 \&$ rerunOrigin $=$ google \& acct $=\mathrm{C} 000037098 \&$ version $=1 \&$ urlVers $\overline{\text { ion }}=0$ \& userid $=6859 \overline{7} 7 \& \mathrm{md} 5=9 \mathrm{f0} \overline{\mathrm{e}} 6951 \mathrm{~d}$ 33b9cf6̈b2a55f88a31f8bc4\&searchtype $=a$ \#bbib001>. Historia Mathematica 33 (2006) 357-358.

8. O'SHEA, Donal. A solução de Poincaré. 1 Ed. São Paulo: Editora Record, 2009. $348 p$.
9. GRAY, Jeremy J.. Non-Euclidean Geometry History - MA290/06V. Disponível em <http://www.ouw.co.uk /bin/ouwsdll.dll?

COURSEMA290_Classical_Studies>. Ed. Open University Learning Resources. London. 1988. ISBN: 9780749229818.

10. VAN GERPEN, Jon. Biodiesel Production. 2002. Disponível em: <http://www.uidaho.edu/bioenergy/bioener gy.ram>. Acesso em: Ago 2007.

11. Silva, Isaac Newton Lima da; De Boni, Luis Alcides Brandini. IBP2145 10 Monitoring the production of biodiesel with real time laser spectroscopy. Disponível em $<$ http://www.ibp.org.br/main.asp? team $=\{d 3595 f 92-$ dae4-46c5-8561639617c6efd9\}>. Acesso em Dez 2010.

12. STEINHAUS, $\quad H$. Mathematical Snapshots, 3rd ed. New York: Dover, p. 251, 1999.

13. HAMILTON, William Rowan. Third Supplement to an Essay on the Ttheory of Systems of Rays. Disponível em: <http://www.maths.tcd.ie/ pub/HistMath/People/Hamilton/Rays/Thrd Sup.pdf>. Acesso em 20 de Maio de 2010. (Transactions of the Royal Irish Academy, vol. 17, part 1 (1837), pp. 1144.)

14. VAN GERPEN, Jon. Biodiesel Production. 2002. Disponível em: <http://www.uidaho.edu/bioenergy/bioener gy.ram>. Acesso em: Ago 2007.

15. TchêQuímica. Aplicativo Biodiesel. Disponível em: < http://www.deboni.he. com.br/ccount/click. php?id=1>. Acesso em: Dez 2010.

16. Wikimidia. PseudoSphere.jpg. Disponí vel em: <http://commons.wikimedia.org /wiki/File:PseudoSphere.jpg>. Acesso em: Dez 2010.

17. Eidt, Caroline. Mais barato e eficaz. Ciência Hoje, vol. 47, n. 277, p. 52-53, 2010. 


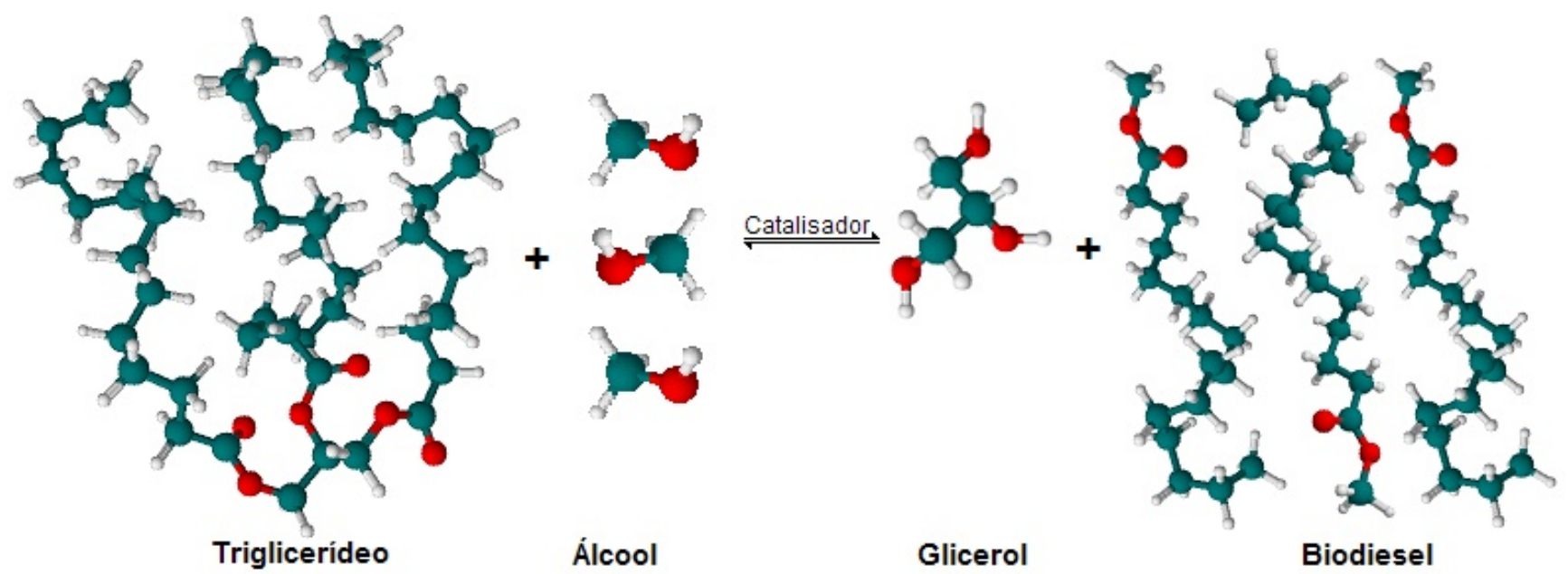

Figura 1. Reação de transesterificação

Vista Superior

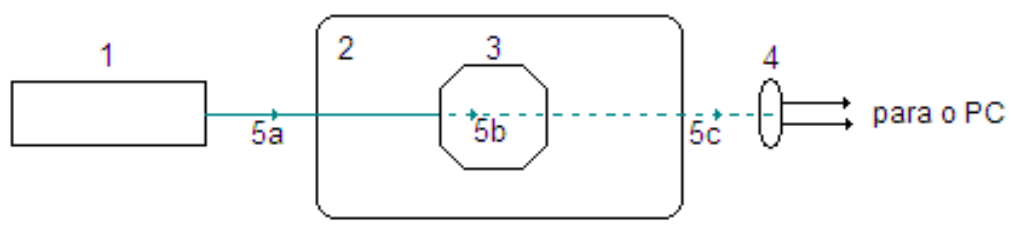

Vista Frontal

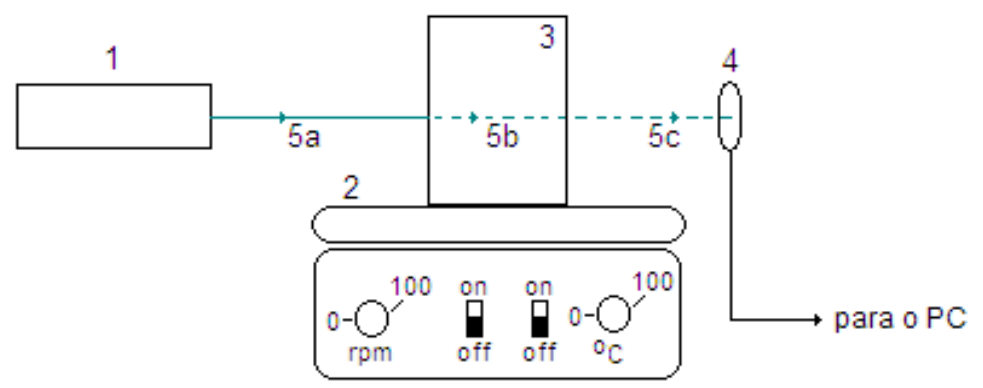

Figura 3. Montagem da reação de transesterificação

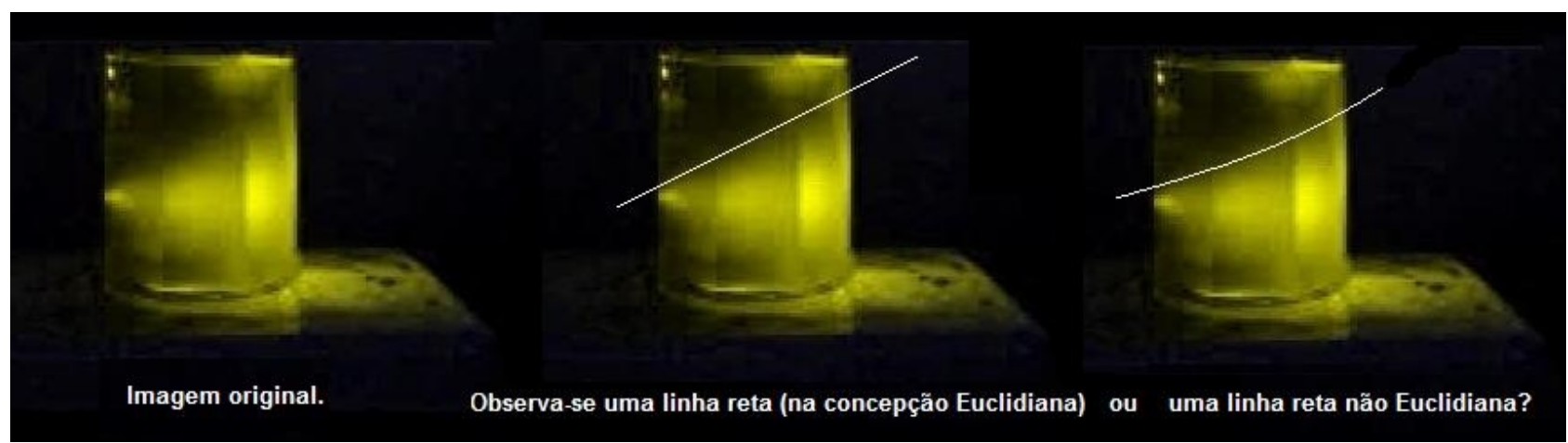

Figura 4. Imagem capturada de um experimento. 


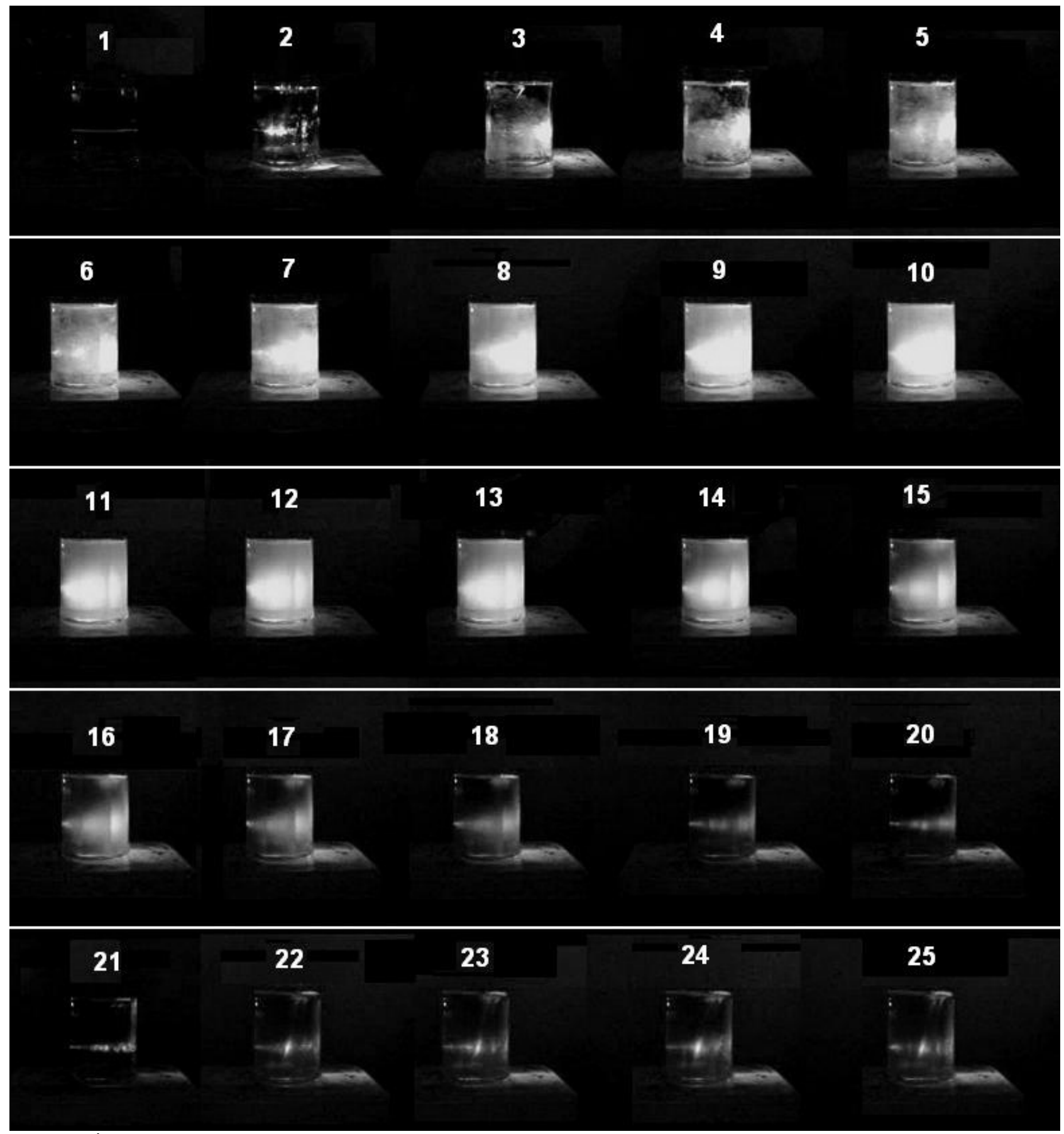

Figura $6^{1}$. Variação da superfície do holograma formado no interior do reator ao longo da reação de transesterificação até o momento do equilíbrio químico. Fonte: Eidt, 2010

1 A Figura 6 foi originalmente cedida à C. Eidt, para publicação na Revista Ciência Hoje, n. 277, Dez. 2010. Para ver esta imagem em cores, favor consultar a revista $\mathrm{CH}$.

Periódico Tchê Química. Vol. 8 - N. 15 - JAN/2011. Porto Alegre - RS. Brasil.

The Periódico Tchê Química (ISSN: 1806-0374; 2179-0302) is an open-access journal since 2004. Journal DOI: 10.52571/PTQ. http://www.tchequimica.com. This text was introduced in this file in 2021 for compliance reasons.

$\odot$ The Author(s)

OPEN ACCESS. This article is licensed under a Creative Commons Attribution 4.0 (CC BY 4.0) International License, which permits use, sharing, adaptation, distribution, and reproduction in any medium or format, as long as you give appropriate credit to the original author(s) and the source, provide a link to the Creative Commons license, and indicate if changes were made. The images or other hird-party material in this article are included in the or exceeds the permitted use, you will need to obtain permission directly from the copyright holder. To view a copy of this license, visit http://creativecommons orgllicenses/by/4.0l. 